\title{
Oral Health and Pregnancy
}

"Recent studies show oral infections might lead to preterm, low-weight births."

\section{What is the public health issue?}

Oral disease, particularly periodontal (gum) disease, may be related to premature and low birth-weight babies.

Pregnant women with periodontal disease may be seven times more likely to have a baby that's born too early and too small, which can result in serious health problems that can be life-long. The exact connection between periodontal disease and preterm birth is unknown, but it might be due to inflammation (swelling) inside the body, the transfer of bacteria from the mouth into the placenta or amniotic fluid, or the release of a particular chemical that stimulates contractions (prostaglandin) from the mouth into the body. Pregnant women should take good care of their teeth and gums by brushing two times every day for two minutes, and flossing daily. They are at an increased risk for gum problems, as almost half of pregnant women in their second or third month of pregnancy get pregnancy gingivitis - this causes gum swelling, pain, and bleeding. In some cases, gums swollen by pregnancy gingivitis can form large growths, called "pregnancy tumors.” They are not cancerous and generally painless. However, if a tumor does not go away, it may need to be removed by a dentist. Pregnant women should also be educated about protecting their new baby's oral health. Bacteria that cause tooth decay can spread from Mother and Child, and this increases the child's risk for tooth decay in the future. Mothers should avoid sharing forks, spoons, and drinks with their babies.

\begin{tabular}{|ll|}
\hline \multicolumn{2}{|c|}{ Oral Health Before and During Pregnancy } \\
$\checkmark$ & Drink fluoridated water \\
$\checkmark$ & Brush your teeth and gums twice a day with a \\
& fluoride toothpaste and floss each day \\
$\checkmark$ & Make an appointment for an exam with a \\
& dentist \\
$\checkmark$ & Make good nutritional choices; limit sweet \\
$\checkmark$ & drinks and avoid sugar \\
$\checkmark$ & Include Vitamin C and Calcium in your daily \\
$\checkmark$ & diet \\
$\checkmark$ & Do not smoke \\
$\checkmark$ & Learn how to take care of the baby's gums and \\
& teeth even before birth \\
\hline
\end{tabular}

Infant and Toddler Oral Health Tips

$\checkmark$ Consult a pediatrician or family physician on the best water to use with infant formula.

$\checkmark$ Start oral care early. Begin by wiping the baby's gums with a cloth or gauze after feeding.

$\checkmark$ Start brushing as soon as the first tooth appears.

$\checkmark$ When the child can brush on his or her own, supervise brushing to make sure fluoride toothpaste is not being used in large amounts and swallowed by the child.

$\checkmark \quad$ Try to schedule the child's first dental visit around his or her first birthday.

\section{Programs at the Massachusetts Department of Public Health}

- The Women, Infant and Children Nutrition Program (WIC) program provides free health education/healthy food to Massachusetts families. To apply for WIC, call 1-800-WIC-1007.

- The Family Planning Program promotes and provides family planning services

- Growing Up Healthy provides information in both English and Spanish about ways to raise a healthy child. This information can be accessed at www.mass.gov

\section{For more information, contact:}

Office of Oral Health

Massachusetts Department of Public Health 250 Washington Street

Boston, MA 02108-4619

Oral.Health@state.ma.us 